\title{
Relayed FSO Communication with Aperture Averaging Receivers and Misalignment Errors
}

\author{
Prabhat Kumar Sharma, Ankur Bansal, Parul Garg, Theodoros A. Tsiftsis, and R. Barrios
}

\begin{abstract}
In this paper, the performance of decode-and-forward relay-assisted free-space-optical (FSO) communication systems under atmospheric turbulence induced fading and misalignment errors is investigated. To mitigate the adverse effects of the atmospheric turbulence, the aperture averaging receivers are considered both at the relay and destination sides. The atmospheric turbulence induced fading is modeled via the exponentiated-Weibull distribution, which has recently been proposed to characterize an FSO link in the presence of finite-sized receiver aperture. The expression for the moment generating function (MGF) of the instantaneous signal-to-noise ratio is derived. Further, new closed form expression for the outage probability is obtained. Moreover, the new expression for the average symbol error rate of the subcarrier intensity modulated M-ary phase shift keying is obtained using MGF-based approach. Finally, numerical examples are discussed and all the derived analytical results are corroborated by Monte-Carlo simulations.
\end{abstract}

\section{INTRODUCTION}

The cooperative free-space optical (FSO) communication has emerged as a potential research trend in last few years. It provides the combined advantages of the wireless optics and co-

Part of this work has been published in proceedings of IEEE International Conference on Communications 2015.

Prabhat Kumar Sharma is with the Department of Electronics and Communication Engineering, Visvesvaraya National Institute of Technology, Nagpur India.(e-mail: prabhatmadhavec1@gmail.com).

Ankur Bansal and Parul Garg are with the Division of Electronics and Communication Engineering, Netaji Subhas Institute of Technology, New Delhi, India. (e-mail: bansal.ankur143@gmail.com, parul_saini@yahoo.co.in).

Theodoros A. Tsiftsis is with the Technological Educational Institute of Central Greece, Lamia 35100, Greece, and with the School of Engineering, Nazarbayev University, Astana 010000, Kazakhstan, (e-mail: tsiftsis@teiste.gr; theodoros.tsiftsis@nu.edu.kz).

R. Barrios is with the Institute of Communications and Navigation, German Aerospace Center (DLR), 82234 Wessling, Germany. (e-mail: Ricardo.Barrios@dlr.de) 
operative communication, which lead to larger bandwidth and improved performance. Several cooperative protocols, such as amplify-and-forward (AF) [1], decode-and-forward (DF) [2], and two-way relaying [3] have been studied recently in the relay-assisted FSO communication. The performance of the above cooperative relaying protocols, however, is highly affected by various perturbations in the propagating laser beam caused by the atmospheric turbulence. The above perturbations include the beam wander that represents the random movement of the instantaneous centre of the beam at receiving aperture. However, as the beam wander-induced fluctuations are very slow, they can be combatted through tracking schemes [4]. Moreover the optical radiation traversing the atmosphere spreads out beyond the diffraction limit of the beam radius, and hence produces the beam spreading [4]. Beam divergence can be minimized by employing a very narrow coherent laser source. In addition, the perturbations in the laser beam produced by the turbulence can cause the scintillation which accounts for the random fluctuations in the beam irradiance. The degrading effects of irradiance fluctuations become more dominant in the FSO systems which employ the point size receive apertures.

The misalignment of laser beam in the receiver plane is another challenge which significantly affects the communication over FSO links, and is a major concern in urban areas where the FSO equipments are mounted on top of high rise buildings. The misalignment errors have been incorporated in the literature as a fading effect. Based on the assumed distribution for the horizontal and vertical displacements in the receiver plane, the misalignment errors are considered to be of two types, zero boresight and non-zero boresight. In zero boresight misalignment, the horizontal and vertical displacements in the receiver planes are characterized as zero mean Gaussian random variable, whereas, the non-zero mean Gaussian distributed horizontal and vertical displacements result into non-zero boresight misalignment. The Rayleigh distribution has been used for modelling the radial displacement in zero boresight [5] misalignment fading, whereas the Rician distribution characterizes the radial displacement in non-zero boresight misalignment [6].

Recently, several techniques have been proposed in the literature [7], [8] to improve the performance of a communication system operating in atmospheric turbulence. Aperture averaging is one of the most widely used alternative technique due to its simplicity and lower cost. In aperture averaging a collecting aperture is placed at the end of the FSO link in the receiver side to combat the adverse effects of the atmospheric turbulence induced fading. The focussing lens 
concentrates the greater portion of the incoming irradiance flux into the photodetector of the point receiver, and hence virtually acts like the finite-sized receiver. The FSO communication system with aperture averaging in lognormal and Gamma-Gamma distributed atmospheric turbulence is analyzed in [9]. In [10], a cooperative FSO system has been studied with AF and DF relaying over lognormal turbulence-induced fading channel which is actually appropriate for weak turbulence conditions. The Authors in [10] considered the intensity modulation directdetection (IM/DD) employing binary pulse position modulation (BPPM). However, it has been shown in [11], [12] that the exponentiated-Weibull (EW) distribution is a more generalized distribution as compared to lognormal and Gamma-Gamma distributions, and provides better fit to simulation and experimental data under all aperture averaging conditions.

The EW distribution [11], [12] assumes that the observed field at the receiver consists of an on-axis component and a weak multipath component which includes several scattered components via different independent off-axis paths. As the number of independent and correlated components in the observed field is unknown, to provide the necessary degrees of freedom to uncorrelated terms, the received irradiance is assumed to be a generalized average of several mutually independent and weighted irradiance random variables. To find the values of parameters appear in EW distribution which provide an excellent fit to all aperture averaging conditions, the probability density function (PDF) is obtained using simulation data for different aperture sizes and turbulence conditions. Thus, the EW distribution captures the effect of aperture-averaging through its constituent parameters as these parameters depend on the scintillation index of the received irradiance. Thus, the EW distribution captures the effect of aperture-averaging through its constituent parameters as these parameters depend on the scintillation index of the received irradiance.

The performance of an FSO communication system over EW channels has been analyzed in several works [13]- [16]. The approximate expressions for the bit error rate (BER) have been derived in [13]- [15]. The average capacity of the optical wireless communication systems over EW distribution turbulence channels has been introduced in [16]. However, the analysis in all above works utilized the PDF of the irradiance, and none of them derived and utilized the PDF of the signal-to-noise ratio (SNR). The statistical analysis through SNR-based approach is less complex and more general as can be extended to different modulation techniques directly. Recently, the performance of a non-cooperative FSO system has been evaluated in terms of the outage 
probability and average BER for various modulation schemes in [17]. The exact expressions for outage probability and the average BER for on-off keying modulation in non-cooperative scenario have been derived in [18] with EW distributed turbulence and non-zero boresight misalignment. Authors in [19] presented the bit-error rate of binary pulse position modulation for multihop DF FSO communication over EW channels with pointing errors. The M-ary phase shift keying (MPSK) along with subcarrier intensity modulation (SIM) has been considered in [20]- [21] for FSO communication systems in Gamma-Gamma atmospheric turbulence. The consideration of SIM facilitates the use of several proven tools and techniques of radio-frequency communication in the analysis of FSO communication systems.

To the best of authors' knowledge the DF cooperative relaying in aperture averaged FSO communication has not been considered over EW distributed turbulence links in the literature. The main contribution of this paper is the performance analysis of a dual-hop DF cooperative system. We extend the analytical framework presented in [17] to the DF cooperative scenario where the source transmits its information to the destination with the help of a DF relay. The detailed contributions through this work are:

- A DF relay assisted FSO communication system with aperture-averaged receivers is studied under the presence of atmospheric turbulence and misalignment errors. In turbulence induced effects, however, only irradiance fluctuations due to scintillation are considered, and the beam wander and beam spreading are ignored.

- New expression for the moment generating function (MGF) of the SNR over EW-distributed atmospheric turbulence channel is derived.

- New expression for the outage probability for the considered DF cooperative system is derived.

- Average symbol error rate (SER) is obtained using the MGF based approach for SIM M-ary phase shift keying (MPSK).

The rest of this paper is organized as follows: A detailed description of channel model is given in section II. In section III, the PDF, CDF and MGF of the instantaneous SNR are derived. Performance analysis metrics such as the outage probability and average SER are obtained in Section IV. Section V provides the numerical results and conclusions are given in section VI. 


\section{SyStem AND CHANNEL MOdEL}

\section{A. Propagation Model and Aperture Averaging}

For characterization of wireless optical links mainly three propagation models have been used in the literature (i) plane-wave propagation model, (ii) spherical-wave propagation model, and (iii) Gaussian beam model. The plane-wave propagation model is suitable for the communication from space to ground whereas the spherical-wave propagation model is used for ground to space communication in absence of the beam wander effect. However, in this paper, we assume that the Gaussian beam model which characterizes the FSO links approximately in terrestrial communication systems.

The spatial coherence of the information carrying Gaussian laser beam reduces when it propagates through the turbulent media. The degree of coherence is measured by the coherence radius of the beam represented as $\rho$. For the $i$ th link, the coherence radius can be given as: $\rho_{0, i}=\left(0.55 C_{n}^{2} \kappa^{2} L_{i}\right)^{-3 / 5}$, where $C_{n}^{2}$ is the refractive index structure parameter (in $\mathrm{m}^{-2 / 3}$ ) and characterizes the strength of atmospheric turbulence, $\kappa$ is the wave number, and $L_{i}$ is the link length.

The aperture averaging is used to reduce the variance of intensity fluctuations at the receiver end. The effect of aperture averaging is quantified by a parameter known as aperture averaging factor, which can be defined as

$$
\mathcal{A}_{i}=\frac{\sigma_{I}^{2}\left(D_{i}\right)}{\sigma_{I}^{2}(0)}, \quad i \in\{1,2\}
$$

where $\sigma_{I}^{2}\left(D_{i}\right)$ represents the variance of intensity fluctuations i.e. scintillation index for a receiver with diameter $D_{i}$ and $\sigma_{I}^{2}(0)$ denotes the scintillation index for a point receiver [8].

Remark 1: The Gaussian beam model has been customarily approximated by spherical-wave model in the literature when the atmospheric conditions makes the beam size at the receiver plane comparatively larger than the collecting aperture size. The aperture averaging factor $\left(\mathcal{A}_{i}\right)$ for spherical beam can also be expressed as [22],

$$
\mathcal{A}_{i}=\left[1+0.33\left(\frac{\kappa D_{i}^{2}}{4 L_{i}}\right)^{\frac{5}{6}}\right]^{-\frac{7}{5}}
$$

where $\kappa$ is the wave number, $D_{1}$ and $D_{2}$ are the diameter of receive apertures at relay and destination, respectively, and $L_{1}$ is the length of source to relay link and $L_{2}$ is the length of relay to destination link. 


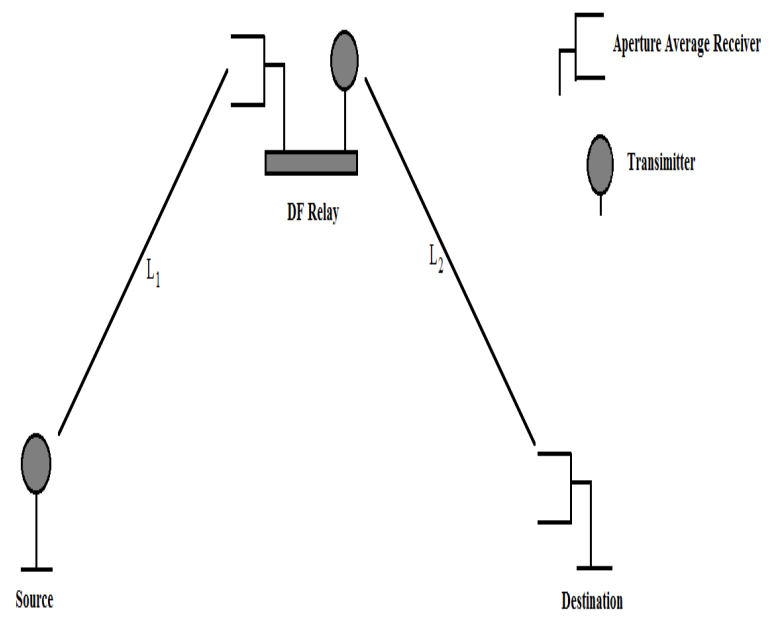

Fig. 1. System Model.

Remark 2: For a point receiver the aperture averaging factor $\mathcal{A}_{i}$ approaches unity, and the aperture diameter is comparable with the atmospheric coherence radius. For the receivers with aperture averaging the receive diameter should considerably be larger than the coherence radius i.e. $D_{i}>>\rho_{0_{i}}$.

\section{B. System Model}

The system model consists of a source, a DF relay, and a destination. The source has one transmit antenna aperture to send its information to the relay. The relay is equipped with one transmit and one receive antenna apertures, and the destination has one receive antenna aperture. The receiving antennas at the relay and the destination have aperture averaged receivers. The diameter of the receive aperture at relay is $D_{1}$ and destination receive aperture diameter is $D_{2}$. It is assumed that the relay is placed in such a way that it can maintain the line-of-sight (LOS) communication with the source as well as with the destination. This kind of cooperative relay placement is practically suitable in the scenarios where either the source and the destination are situated at a long distance apart or they are at non-LOS from each other. 


\section{Channel Model}

We consider a composite model for the FSO channels between the source and the relay, represented by the channel coefficient $\mathcal{H}_{1}$, and between the relay and the destination, represented by the channel coefficient $\mathcal{H}_{2}$. The channel coefficient $\mathcal{H}_{i}$, where $i \in\{1,2\}$, is composed of atmospheric turbulence induced fading represented by the coefficient $h_{a t_{i}}$, and the misalignment fading denoted as $h_{m_{i}}$. Thus composite channel coefficient can be given as

$$
\mathcal{H}_{i}=h_{a t_{i}} h_{m_{i}} .
$$

The atmospheric turbulence induced fading is modelled under EW distribution. The PDF of the coefficient $h_{a t_{i}}\left(h_{a t_{i}}>0\right)$, is given by [11]

$$
f_{h_{a_{i}}}\left(h_{a t_{i}}\right)=\frac{\alpha_{i} \beta_{i}}{\eta_{i}}\left(\frac{h_{a t_{i}}}{\eta_{i}}\right)^{\beta_{i}-1} \exp \left[\left(\frac{h_{a t_{i}}}{\eta_{i}}\right)^{\beta_{7}}\right]\left\{1-\exp \left[-\left(\frac{h_{a t_{i}}}{\eta_{i}}\right)^{\beta_{i}}\right]\right\}^{\alpha_{i}-1},
$$

where $\beta_{i}>0$ and $\alpha_{i}>0$ are the shape parameters, and $\eta_{i}>0$ is the scale parameter of the turbulence channel represented by the channel coefficient $h_{a t_{i}}$.

The EW distribution incorporates the aperture averaging through its parameters as the parameters depend on the scintillation index (and hence on the dimension of receive aperture and atmospheric turbulence condition of the link) [23] as per the following relation,

$$
\begin{aligned}
\alpha_{i} & \simeq \frac{7.22 \sigma_{I}^{\frac{2}{3}}\left(D_{i}\right)}{\Gamma\left(2.487 \sigma_{I}^{\frac{2}{6}}\left(D_{i}\right)-0.104\right)} \\
\beta_{i} & \simeq 1.012\left(\alpha_{i} \sigma_{I}^{2}\left(D_{i}\right)\right)^{-\frac{13}{25}}+0.142, \\
\eta_{i} & =\frac{1}{\alpha_{i} \Gamma\left(1+\frac{1}{\beta_{i}}\right) \xi\left(\alpha_{i}, \beta_{i}\right)}, \text { where } \\
\xi\left(\alpha_{i}, \beta_{i}\right) & =\sum_{j=0}^{\infty} \frac{(-1)^{j} \Gamma\left(\alpha_{i}\right)}{j !(1+j)^{1+\frac{1}{\beta_{i}}} \Gamma\left(\alpha_{i}-j\right)}
\end{aligned}
$$

Remark 3: The parameter definition in (5) [23, Eq 21] has not been tested experimentally for weak turbulence channel conditions, however, the validity of EW parameters in all turbulence conditions (including the weak atmospheric turbulence) has been confirmed through best fitting process using the Levenberg-Marquardt least-square algorithm [23, section 5]. Moreover, the parameter definition in (5) has been used widely in [31, section 4.5.6] for the purpose of performance evaluation of FSO communication systems in all atmospheric turbulence scenarios.

The misalignment fading $h_{m_{i}}$ has been characterized statistically in [5]. When horizontal and vertical jitter in the receiver plane are independent of each other, and are distributed with identical 
zero mean Gaussian random variable, then the radial displacement at the receiver follows the Rayleigh distribution. Hence the PDF of $h_{m_{i}}$ can be given, as

$$
f_{h_{m_{i}}}\left(h_{m_{i}}\right)=\frac{\mu_{i}^{2}}{A_{0_{i}}^{\mu_{i}^{2}}} h_{m_{i}}^{\mu_{i}^{2}-1}, \quad 0 \leq h_{m_{i}} \leq A_{0_{i}}
$$

where, for the misalignment errors corresponding to channel $\mathcal{H}_{i}$, the term $A_{0_{i}}=\left[\operatorname{erf}\left(\nu_{i}\right)\right]^{2}$

represents the fraction of the collected optical power, $\nu_{i}=\sqrt{\pi a_{i}^{2} / 2 w_{b_{i}}^{2}}, a_{i}$ is the radius of receiver aperture, and $w_{b_{i}}$ is normalized beam waist. Further, $\mu_{i}=\frac{\omega_{i}}{2 \sigma_{s_{i}}}, \omega_{i}$ is the equivalent beam width at the receiver and can be given as $\omega_{i}=\left[\sqrt{A_{0, i} \pi} w_{b_{i}}^{2} /\left(2 \nu_{i} \exp \left(-\nu_{i}^{2}\right)\right)\right]^{1 / 2}$, and $\sigma_{s_{i}}^{2}$ is the variance of pointing error displacement characterized by the horizontal sway and elevation [5].

\section{Transmission Protocol}

The DF transmission protocol, which takes place in two time slots, is considered. In the first time slot, the source transmits its information to the relay, and the relay receives the transmission at its receiving antenna aperture. The relay then decodes the information received, and after decoding, forwards the decoded signal to the destination, in second time slot.

The received signal at relay in first time slot is

$$
y_{r}=\mathcal{H}_{1} \mathcal{R} x+n_{r}
$$

where $x$ is the transmitted symbol from source, $\mathcal{R}$ is the responsivity of the photodetector, $n_{r}$ is additive white Gaussian noise with zero mean and $N_{0}$ variance at the relay.

Similarly, the signal received at the destination in second time slot

$$
y_{d}=\mathcal{H}_{2} \mathcal{R} \hat{x}+n_{d}
$$

where $\hat{x}$ is the decoded version of $x$ and $n_{d}$ is additive white Gaussian noise with zero mean and $N_{0}$ variance at destination. Without loss of generality we assume that responsivity is same for the photodetectors at both relay and destination.

\section{STATISTICS OF THE INSTANTANEOUS SNR}

In this section we derive the PDF, CDF and MGF of the instantaneous SNR over EWdistributed FSO channels. The SNR over channel $\mathcal{H}_{i}$ is given by $\gamma_{i}=\bar{\gamma}_{0}\left|\mathcal{H}_{i}\right|^{2}$, where $\bar{\gamma}_{0}=$ 
$\frac{\left(P_{t} \mathcal{R} \zeta\right)^{2}}{N_{0}}$. The term $P_{t}$ is the average transmit power, $N_{0}$ is the average noise power of the additive white Gaussian noise at the receiver, and $\zeta$ is the modulation index [13, eq. (3)]. The average SNR can be given as $\bar{\gamma}_{i}=\bar{\gamma}_{0} E\left[\left|\mathcal{H}_{i}\right|^{2}\right]$, where $E[\cdot]$ is the expectation operator [24].

Theorem 1. The PDF of the instantaneous $S N R \gamma_{i}$ can be given by

$$
f_{\gamma_{i}}\left(\gamma_{i}\right)=B_{1_{i}} \sum_{j=0}^{\infty} \Psi_{i}(j) \gamma_{i}^{\frac{\mu_{i}^{2}}{2}-1} \Gamma\left[\tau_{i}, B_{2_{i}}(j) \gamma_{i}^{\frac{\beta_{i}}{2}}\right],
$$

where $\Psi_{i}(j)=\frac{(-1)^{j} \Gamma\left(\alpha_{i}\right)}{j ! \Gamma\left(\alpha_{i}-j\right)(1+j)^{1-\frac{\mu_{i}^{2}}{\beta_{i}}}}, B_{1_{i}}=\frac{\alpha_{i} \mu_{i}^{2}}{2\left(\eta A_{0_{i}} \sqrt{\bar{\gamma}_{0}}\right)^{\mu_{i}^{2}}}, \tau_{i}=1-\frac{\mu_{i}^{2}}{\beta_{i}}$, and $B_{2_{i}}(j)=\frac{1+j}{\left(\eta_{i} A_{0_{i}} \sqrt{\left.\overline{\gamma_{0}}\right)^{\beta_{i}}}\right.}, \Gamma(\cdot)$ is the gamma function, and $\Gamma(\cdot, \cdot)$ is the upper incomplete gamma function [25]

Proof. Proof of (9) is given in Appendix I. Moreover, the validity of the PDF defined in (9) is proved in Appendix II.

The expression of the PDF in (9) consists of infinite summation which results from the use of Newton's generalized binomial expansion, however it can easily be verified using MATLAB or MATHEMATICA that the infinite summation is convergent and ten to fifteen terms are sufficient for this series to converge [14]. Further this can also be verified from Fig. 2, where we plotted the PDF for $j=0$ to 10 , and $j=0$ to 100 terms, and it can be observed that the plots of the PDF in both the cases are perfectly matched.

Lemma 1. The CDF of received SNR can be given as,

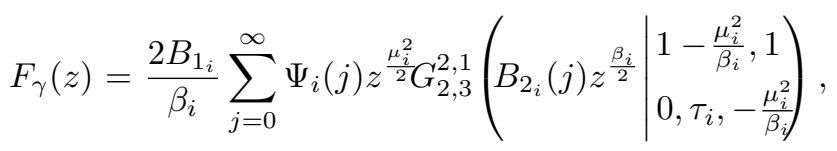

Proof. The CDF of the SNR is defined as,

$$
\begin{aligned}
F_{\gamma_{i}}(z) & =\int_{0}^{z} f_{\gamma_{i}}\left(\gamma_{i}\right) d \gamma_{i} \\
& =B_{1_{i}} \sum_{j=0}^{\infty} \Psi_{i}(j) \int_{0}^{z} \gamma_{i}^{\frac{\mu_{i}^{2}}{2}-1} \Gamma\left[\tau_{i}, B_{2_{i}}(j) \gamma_{i}^{\frac{\beta_{i}}{2}}\right] .
\end{aligned}
$$

On replacing the upper incomplete gamma function in (11) with its Meijer's-G equivalent [26, eq. (06.06.26.0005.01)] and substituting $\gamma_{i}^{\frac{\beta_{i}}{2}}=t$ we get,

$$
F_{\gamma}(z)=\frac{2 B_{1_{i}}}{\beta_{i}} \sum_{j=0}^{\infty} \Psi_{i}(j) \int_{0}^{z^{\frac{\beta_{i}}{2}}} t^{\frac{\mu_{i}^{2}}{\beta_{i}}-1} G_{1,2}^{2,0}\left(B_{2_{i}}(j) t \mid \begin{array}{c}
1 \\
0, \tau_{i}
\end{array}\right) d t,
$$

where $G_{p q}^{m, n}\left(\left.\cdot\right|^{\cdot}\right)$ is the Meijer's $\mathrm{G}$ function [26, eq. (07.34.02.0001.01)]. Using [26, eq. (07.34.21.0084.01)] the integral in (12) can be reduced to (10). 


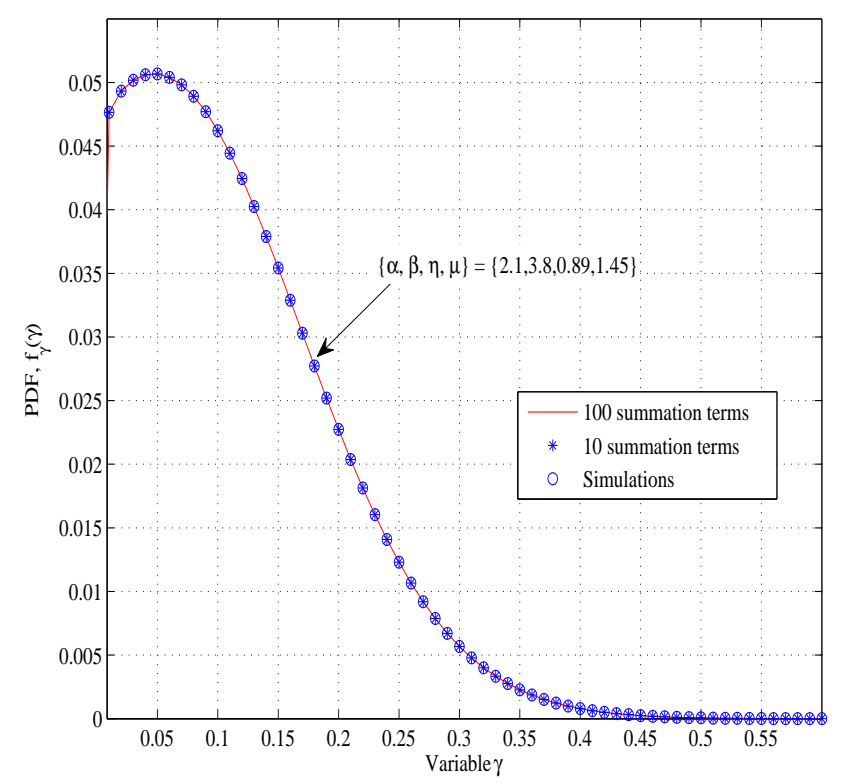

Fig. 2. The PDF of the instantaneous SNR $\gamma_{i}$ given in (9) .

Lemma 2. MGF of the instantaneous received $S N R$ can be given as

$$
\begin{aligned}
\mathcal{M}_{\gamma_{i}}(s) & =B_{1, i} \sum_{j=0}^{\infty} \Psi_{i}(j) \frac{k_{i}^{\tau_{i}-1 / 2} l_{i}^{\frac{\mu_{i}^{2}-1}{2}} s^{-\frac{\mu_{i}^{2}}{2}}}{(2 \pi)^{\frac{l_{i}+2 k_{i}-3}{2}}} \\
& \times G_{l_{i}+k_{i}, 2 k_{i}}^{2 k_{i}, 2 l_{i}}\left(\frac{\left(B_{2_{i}}(j)\right)^{k_{i}} l_{i}^{l_{i}}}{s^{l_{i}} k_{i}^{k_{i}}} \mid \begin{array}{r}
\Delta\left(l_{i}, 1-\frac{\mu_{2}}{2}\right), \Delta\left(k_{i}, 1\right) \\
\Delta\left(k_{i}, \tau_{i}\right) \Delta\left(k_{i}, 0\right)
\end{array}\right),
\end{aligned}
$$

where $l_{i}$ and $k_{i}$ are integer constants so that $\beta_{i}=\frac{l_{i}}{k_{i}}$, and $\Delta(m, n)=(n / m)((n+1) / m) \ldots((n+$ $m-1) / m)$.

Proof. The MGF of the SNR is defined as $\mathcal{M}_{\gamma_{i}}(s)=\int_{0}^{\infty} f_{\gamma_{i}}\left(\gamma_{i}\right) \exp \left(-s \gamma_{i}\right) d \gamma_{i}$. Using (9), [26, eq. (06.06.26.0005.01)] and [27, eq. (2.24.1.1)] the $\operatorname{MGF,} \mathcal{M}_{\gamma_{i}}(s)$ can be written as given in (13).

\section{PERformanCE ANALYSis}

\section{A. Outage Analysis}

We now analyze the outage behavior of the given dual hop DF communication system over EW-distributed FSO channels. The outage event is said to be occurred when received instantaneous SNR falls below some specified threshold SNR. For the dual hop DF relaying 
system, the outage occurs if either source to relay or relay to destination link falls in outage. Thus the outage probability in terms of received instantaneous SNRs can be given as,

$$
P_{\text {out }}=\operatorname{Pr}\left(\gamma_{1}<\gamma_{t h}\right)+\left(1-\operatorname{Pr}\left(\gamma_{1}<\gamma_{t h}\right)\right) \operatorname{Pr}\left(\gamma_{2}<\gamma_{t h}\right)
$$

where $\gamma_{t h}$ is the threshold SNR and $\gamma_{1}$ and $\gamma_{2}$ are the received instantaneous SNRs over channels $\mathcal{H}_{1}$ and $\mathcal{H}_{2}$, respectively.

The probability terms $\operatorname{Pr}\left(\gamma_{1}<\gamma_{t h}\right)$ and $\operatorname{Pr}\left(\gamma_{2}<\gamma_{t h}\right)$ can be evaluated using (10). Thus, the expression of outage probability can be given by substituting (10) into (14) as,

$$
P_{\text {out }}=F_{\gamma_{1}}\left(\gamma_{t h}\right)+\left(1-F_{\gamma_{1}}\left(\gamma_{t h}\right)\right) F_{\gamma_{2}}\left(\gamma_{t h}\right)
$$

\section{B. Average SER for MPSK}

The equal energy MPSK constellation is considered where transmission of each symbol is equiprobable. In SIM, the signal $z(t)$ is represented in terms of its in-phase component $z_{I}(t)$ and quadrature component $z_{Q}(t)$ i.e. $z(t)=z_{I}(t)+j z_{Q}(t)$, where $z_{I}(t)=\sum_{n=0}^{\infty} x_{I}[n] g\left(t-n T_{s}\right)$ and $z_{Q}(t)=\sum_{n=0}^{\infty} x_{Q}[n] g\left(t-n T_{s}\right)$, respectively; $g(t)$ represents the pulse shaping function and $T_{s}$ is the symbol duration. Further, $x_{I}[n]$ and $x_{Q}[n]$ are the in-phase and quadrature components of the $n$-th data symbol $x[n]$, and can be written as $x_{I}[n]=\cos \phi_{n}$ and $x_{Q}[n]=\sin \phi_{n}$, respectively, where $\phi_{n} \in\left\{\theta, \theta+\frac{2 \pi}{M}, \ldots, \theta+\frac{2(M-1) \pi}{M}\right\}$ and $\theta \in\{0,2 \pi\}$.

For a symmetric MPSK constellation with equidimensional decision regions, the probability of error $P_{S R}^{M P S K}(e)$ can be given as [28], [29]

$$
P_{S R}^{M P S K}(e)=1-\sum_{k=1}^{M} P_{k}\left(\gamma_{1}\right) P_{k}\left(\gamma_{2}\right)
$$

where $P_{k}\left(\gamma_{1}\right)$ is the probability that the source transmits $x_{j}$ and the relay receives it as $x_{k}$ i.e. $P_{k}\left(\gamma_{1}\right)=\operatorname{Pr}\left[\mathcal{H}_{1} x_{j}+N_{R} \in \mathcal{D}_{k}\right]$ and $P_{k}\left(\gamma_{2}\right)$ is the probability that the relay transmits $x_{k}$ and it is received at the destination as $x_{j}$ i.e. $P_{k}\left(\gamma_{2}\right)=\operatorname{Pr}\left[\mathcal{H}_{2} s_{k}+N_{B} \in \mathcal{D}_{j}\right], j, k \in\{1,2, \ldots, M\}, j \neq k$, $\mathcal{D}_{j}$ and $\mathcal{D}_{k}$ are the decision regions coresponding to symbol $x_{j}$ and $x_{k}$, respectively, and the terms $N_{R}$ and $N_{B}$ represent the AWGN with zero mean and variance $N_{0}$ at the relay and the destination, respectively. 
The probabilities $P_{k}\left(\gamma_{1}\right)$, and $P_{k}\left(\gamma_{2}\right)$, can be given as [28]

$$
P_{k}\left(\gamma_{i}\right)= \begin{cases}1-\frac{1}{\pi} \int_{0}^{\frac{(M-1) \pi}{M}} \mathcal{M}_{\gamma_{i}}\left(\frac{\sin ^{2}\left(\frac{\pi}{M}\right)}{\sin ^{2}(\phi)}\right) d \phi & k=1 \\ \frac{1}{\pi} \int_{0}^{\frac{(M-1) \pi}{M}} \mathcal{M}_{\gamma_{i}}\left(\frac{\sin ^{2}\left(\frac{\pi}{M}\right)}{\sin ^{2}(\phi)}\right) d \phi & k=\frac{M}{2}+1 \\ \frac{1}{2 \pi} \int_{0}^{\pi-a_{k-1}} \mathcal{M}_{\gamma_{i}}\left(\frac{\sin ^{2}\left(a_{k-1}\right)}{\sin ^{2}(\phi)}\right) d \phi & \\ -\frac{1}{2 \pi} \int_{0}^{\pi-a_{k}} \mathcal{M}_{\gamma_{i}}\left(\frac{\sin ^{2}\left(a_{k}\right)}{\sin ^{2}(\phi)}\right) d \phi & \text { otherwise }\end{cases}
$$

where $i \in\{1,2\}$.

Remark 4: The occurrence of error for the DF cooperative strategy, as given in (16), can be explained as follows: suppose source transmits the symbol $x_{j} \in \mathcal{A}$, where $\mathcal{A}$ is an arbitrary M-PSK constellation. Now the relay erroneously decodes it as another symbol $x_{k}, k \neq j$ and $j, k \in\{1,2, \ldots, M\}$. Now it is quite possible that destination erroneously decides the symbol $x_{k}$ as $x_{j}$. In this way the error made by the relay is compensated by another error made by the destination, and hence overall transmission becomes error free.

The integrals of the form $\frac{1}{\pi} \int_{0}^{\Theta} \mathcal{M}_{\gamma_{i}}\left(\frac{g}{\sin ^{2}(\phi)}\right) d \phi$ can be accurately approximated as [30],

$$
\begin{aligned}
\frac{1}{\pi} \int_{0}^{\Theta} \mathcal{M}_{\gamma_{i}}\left(\frac{g}{\sin ^{2}(\phi)}\right) d \phi & \approx\left(\frac{\Theta}{2 \pi}-\frac{1}{6}\right) \mathcal{M}_{\gamma_{i}}(g)+\frac{1}{4} \mathcal{M}_{\gamma_{i}}\left(\frac{4 g}{3}\right) \\
& +\left(\frac{\Theta}{2 \pi}-\frac{1}{4}\right) \mathcal{M}_{\gamma_{i}}\left(\frac{g}{\sin ^{2} \Theta}\right)
\end{aligned}
$$

where $\Theta \in\left\{\frac{(M-1) \pi}{M}, \pi-a_{k-1}, \pi-a_{k}\right\}$, and $g \in\left\{\sin ^{2}\left(\frac{\pi}{M}\right), \sin ^{2}\left(a_{k-1}\right), \sin ^{2}\left(a_{k}\right)\right\}$.

The probability term $P_{k}\left(\gamma_{i}\right)$ as defined in (17), can be rewritten using (18) as follows,

$$
P_{k}\left(\gamma_{i}\right) \approx \begin{cases}1-\left[c_{1} \mathcal{M}_{\gamma_{i}}\left(\sin ^{2}\left(\frac{\pi}{M}\right)\right)+\frac{1}{4} \mathcal{M}_{\gamma_{i}}\left(\frac{4 \sin ^{2}\left(\frac{\pi}{M}\right)}{3}\right)\right. & \\ \left.+c_{2} \mathcal{M}_{\gamma_{i}}\left(\frac{\sin ^{2}\left(\frac{\pi}{M}\right)}{\sin ^{2} \phi_{2}}\right)\right] & k=1 \\ c_{1} \mathcal{M}_{\gamma_{i}}\left(\sin ^{2}\left(\frac{\pi}{M}\right)\right)+\frac{1}{4 M} \mathcal{M}_{\gamma_{i}}\left(\frac{4 \sin ^{2}\left(\frac{\pi}{M}\right)}{3}\right) & \\ +c_{2} \mathcal{M}_{\gamma_{i}}\left(\frac{\sin ^{2}\left(\frac{\pi}{M}\right)}{\sin ^{2} \phi_{2}}\right) & k=\frac{M}{2}+1 \\ \frac{1}{2}\left[c_{3} \mathcal{M}_{\gamma_{i}}\left(\sin ^{2} \phi_{1}\right)+\frac{1}{4} \mathcal{M}_{\gamma_{i}}\left(\frac{4 \sin ^{2} \phi_{1}}{3}\right)\right. & \\ +c_{4} \mathcal{M}_{\gamma_{i}}\left(\frac{\sin ^{2} \phi_{1}}{\sin ^{2} \phi_{2}}\right)-c_{6} \mathcal{M}_{\gamma_{i}}\left(\sin ^{2} \phi_{0}\right) & \\ \left.+\frac{1}{4} \mathcal{M}_{\gamma_{i}}\left(\frac{4 \sin ^{2} \phi_{0}}{3}\right)+c_{5} \mathcal{M}_{\gamma_{i}}\left(\frac{\sin ^{2} \phi_{0}}{\sin ^{2} \phi_{2}}\right)\right] & \text { otherwise }\end{cases}
$$

where $c_{1}=\left(\frac{2 M-3}{6 M}\right), c_{2}=\left(\frac{M-1}{4 M}\right), c_{3}=\left(\frac{2 \pi-3 a_{k-1}}{6 \pi}\right), c_{4}=\left(\frac{\pi-2 a_{k-1}}{4 \pi}\right), c_{5}=\left(\frac{\pi-2 a_{k}}{4 \pi}\right), c_{6}=\left(\frac{2 \pi-3 a_{k}}{6 \pi}\right)$, $\phi_{0}=\pi-a_{k} \phi_{1}=\pi-a_{k-1}$, and $\phi_{2}=\left(\frac{(M-1) \pi}{M}\right)$.

The new expression of the average SER $P_{S R}^{M P S K}(e)$ for MPSK signalling can be derived by substituting (19) in (16). 


\section{Numerical Results}

In this section, numerical results are derived using Monte-Carlo simulations. Specifically, the simulation of EW channels is implemented by following the same approach as the one presented in [23], [31]. An EW random variable with parameter $\{\alpha, \beta, \eta\}$ is approximately equal to the random variable obtained from $\max \left(w_{1}, w_{2}, \ldots w_{m}\right)$, where all $w_{i}\{i=1,2, . . m\}$, are identical Weibull distributed random variables with parameters $\{\beta, \eta\}$ and $m$ is the nearest integer to $\alpha$.

To evaluate the effect of aperture averaging in different atmospheric turbulence conditions, three different values of refractive index structure parameter $C_{n}^{2}$ are considered i.e. $C_{n}^{2}=$ $7.2 \times 10^{-15} \mathrm{~m}^{-2 / 3}$ for weak turbulence, $C_{n}^{2}=5.0 \times 10^{-14} \mathrm{~m}^{-2 / 3}$ for moderate turbulence and $C_{n}^{2}=3.6 \times 10^{-13} \mathrm{~m}^{-2 / 3}$ for strong turbulence conditions. The wavelength of optical signals is taken as $780 \mathrm{~nm}$. The link lengths are considered to be $1 \mathrm{~km}$ i.e. $L_{1}=L_{2}=1 \mathrm{~km}$. For the assumption of spherical wave the coherence radius and Rytov variances are calculated as $\rho_{0, i}=\left(0.55 C_{n}^{2} \kappa^{2} L_{i}\right)^{-3 / 5}$ and $\sigma_{r v}^{2}=1.23 C_{n}^{2} \kappa^{\frac{7}{6}} L_{i}^{\frac{11}{6}}$. Thus for weak turbulence $\rho_{0,1}=\rho_{0,2}=3.58$ $\mathrm{cm}, \sigma_{r v}^{2}=0.3194$; for moderate turbulence $\rho_{0,1}=\rho_{0,2}=1.112 \mathrm{~cm}, \sigma_{r v}^{2}=2.2181$, and for strong turbulence $\rho_{0,1}=\rho_{0,2}=0.34 \mathrm{~cm}, \sigma_{r v}^{2}=15.97$. The misalignment fading is characterized as follows: the beam radius at receiver $w_{b_{1}}=w_{b_{2}}=2 \mathrm{~m}$, aperture radius $a_{i}=\frac{D_{i}}{2}$. Moreover, without loss of generality we consider $D_{1}=D_{2}=D, \rho_{0,1}=\rho_{0,2}=\rho, A_{0_{1}}=A_{0_{2}}=A_{0}$ and $\sigma_{s_{1}}=\sigma_{s_{2}}=\sigma_{s}$. Additionally, for numerical analysis purpose (for Fig. 3, Fig. 4 and Fig. 5 ) we consider that the source to relay and relay to destination channels are identical i.e. $\beta_{1}=\beta_{2}=\beta$, $\alpha_{1}=\alpha_{2}=\alpha, \eta_{1}=\eta_{2}=\eta$. The case of non-identical $s-r$ and $r-d$ links is discussed in Fig. 6.

In Fig. 3, the outage probability is plotted for different values of aperture averaging diameter considering moderate atmospheric turbulence regime and $\sigma_{s}=30 \mathrm{~cm}$. Three aperture diameters are considered (i.e. $D=200 \mathrm{~mm}, 100 \mathrm{~mm}, 50 \mathrm{~mm}$ ) along with the case when there is no aperture averaging (when the point receiver is deployed). The threshold SNR for the plot is taken as $\gamma_{t h}=2 \mathrm{~dB}$. For point receivers, values of the parameters $\{\alpha, \beta, \eta\}$ are obtained using [31, Ch. 2 Eq. 59] and value of Rytov variance corresponding to the given turbulence scenario. It can be observed from this figure that the aperture averaging significantly improves the system performance as compared to the case of point receivers. Moreover, as the diameter of receive aperture is increased, the outage probability deteriorates further. 
The outage behaviour of the considered dual-hop DF system for aperture averaging effect in different fading situations can be observed in Fig. 4. Here the outage probability is plotted against the average SNR for four different values of aperture averaging diameter (point receiver, $D=200 \mathrm{~mm}, 100 \mathrm{~mm}, 50 \mathrm{~mm}$ ) in weak and strong atmospheric turbulence conditions. For misalignment fading we consider $\sigma_{s}=20 \mathrm{~cm}$. It can be seen from this figure that the outage performance is intuitively better in lower atmospheric turbulence, and it improves further as the size of receive aperture is increased from point to $D=200 \mathrm{~mm}$. Further, it can be observed from this plot that the shift in the outage probability curves for point aperture to the $50 \mathrm{~mm}$ diameter aperture is more significant than the shift in the curves from $50 \mathrm{~mm}$ diameter to 100 $\mathrm{mm}$ diameter or from $100 \mathrm{~mm}$ diameter to $200 \mathrm{~mm}$ diameter apertures. Additionally, it can also be seen that the improvement in the system outage performance with respect to the atmospheric turbulence conditions, is more prominent for $D=50 \mathrm{~mm}$. It indicates the existence of some receive aperture size (say $D_{\text {optimum }}$ ), for a given turbulence and misalignment conditions, which limits the further improvement in the performance.

The effect of receive aperture diameter and misalignment fading is observed on the SER performance for QPSK and 8-PSK signalling schemes in Fig. 5. We consider two possibilities of receiver, one the point receiver and the other is receiver with $50 \mathrm{~mm}$ aperture diameter. Two values for misalignment jitter standard deviation $\sigma_{s}$ are considered i.e. $\sigma_{s}=20 \mathrm{~mm}$ and $\sigma_{s}=40$ $\mathrm{mm}$. It should be noted that higher jitter standard deviation indicates severity of misalignment. The SER performance shown in this figure reveals that the reduction in the jitter standard deviation improves the performance for both point receiver and aperture averaged receivers. However, it can be observed that change in the performance with misalignment errors is less significant for point receivers as compared to $50 \mathrm{~mm}$ diameter receiver. This is because of the fact that the intensity of information carrying laser beam collected by the point receiver is heavily affected by even smaller misalignment errors, however the small misalignment in the receiver plane can be compensated by aperture averaged receiver so as to facilitate larger collection of optical power in the receiver.

The effect of non-identical $s-r$ and $r-d$ links in weak and strong turbulence scenarios is shown in Fig 6, where the average error rate for binary phase shift keying (BPSK) modulation is plotted with the average SNR $\left(\bar{\gamma}_{r d}\right)$ of $r-d$ link. The average SNR $\left(\bar{\gamma}_{s r}\right)$ of $s-r$ link is taken as, $\bar{\gamma}_{s r}=\bar{\gamma}_{s r}-5 \mathrm{~dB}, \bar{\gamma}_{s r}=\bar{\gamma}_{s r}$, and $\bar{\gamma}_{s r}=\bar{\gamma}_{s r}+5 \mathrm{~dB}$. The $D$ is considered to be $50 \mathrm{~mm}$. 


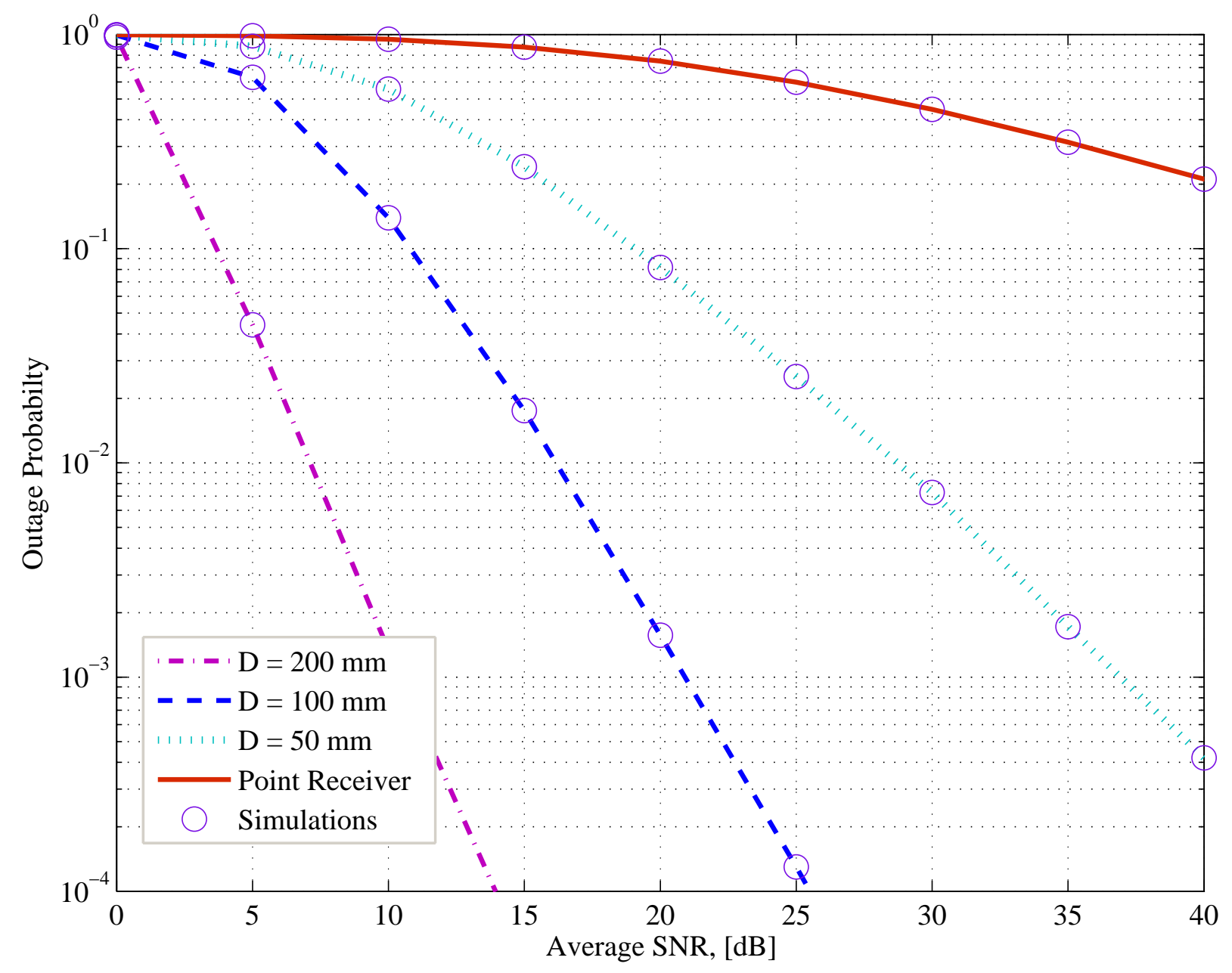

Fig. 3. Outage probability for different aperture averaging conditions.

The increase in $\bar{\gamma}_{s r}$ improves the error performance for strong as well as weaker atmospheric turbulence conditions. Moreover in weaker turbulence, the betterment in the $s-r$ link statistics results in more better performance as compared to that in stronger turbulence.

\section{CONCLUSIONS}

In this paper, the performance of an aperture averaging-based DF cooperative communication system is investigated over EW distributed FSO channels. The PDF, CDF and MGF of the SNR, over the composite channel which includes turbulence induced fading, and misalignment fading, were derived. Using the statistics of the SNR, closed form expressions for the outage probability and average SER were obtained for SIM-MPSK modulation. 


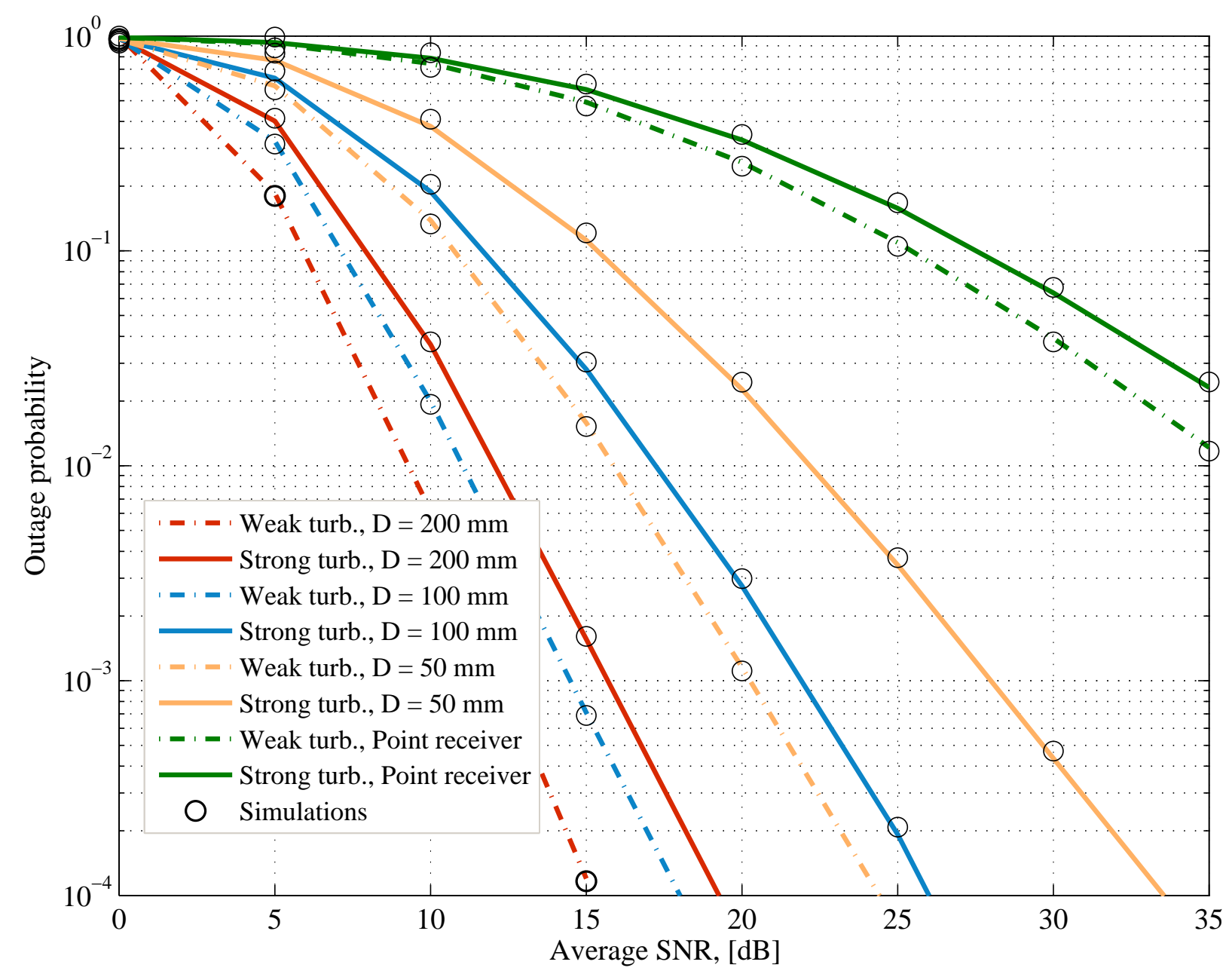

Fig. 4. Outage probability for different turbulence and aperture averaging conditions.

It was shown that for a given aperture size, the turbulence induced fading is more dominant in lower misalignment, and at high misalignment fading the effect of turbulence induced fading becomes less severe. Further, the performance of FSO communication system in atmospheric turbulence can be improved using the aperture averaging. In moderate misalignment and high turbulence induced fading it was observed that any increase in receive aperture diameter significantly reduces the error probability. 


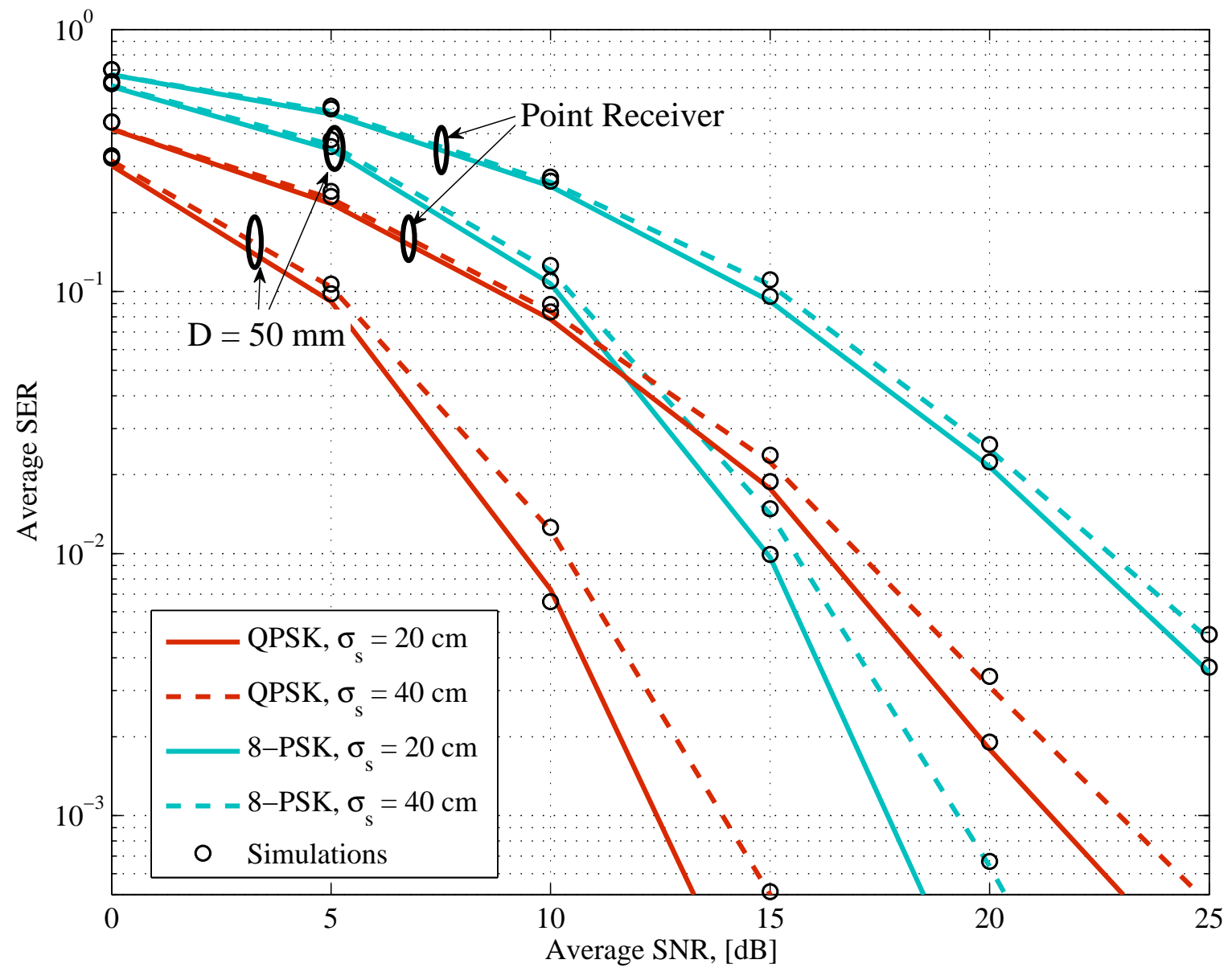

Fig. 5. Average BER for MPSK and 8PSK in varying turbulence and misalignment errors.

\section{ProOf OF THEOREM 1}

In (3), the terms $h_{a t_{i}}$ and $h_{m_{i}}$ are random variables with PDFs given in (4) and (6), respectively, and the term is the deterministic factor. To derive the PDF of $\mathcal{H}_{i}$, we first obtain the PDF of the RV $X \triangleq h_{a t_{i}} h_{m_{i}}$. The PDF of $X$ can be written as,

$$
f_{X}(x)=\int_{\frac{x}{A_{0_{i}}}}^{\infty} f_{h_{m_{i}}}\left(x \mid h_{a t_{i}}\right) f_{h_{a t_{i}}}\left(h_{a t_{i}}\right) d h_{a t_{i}} .
$$




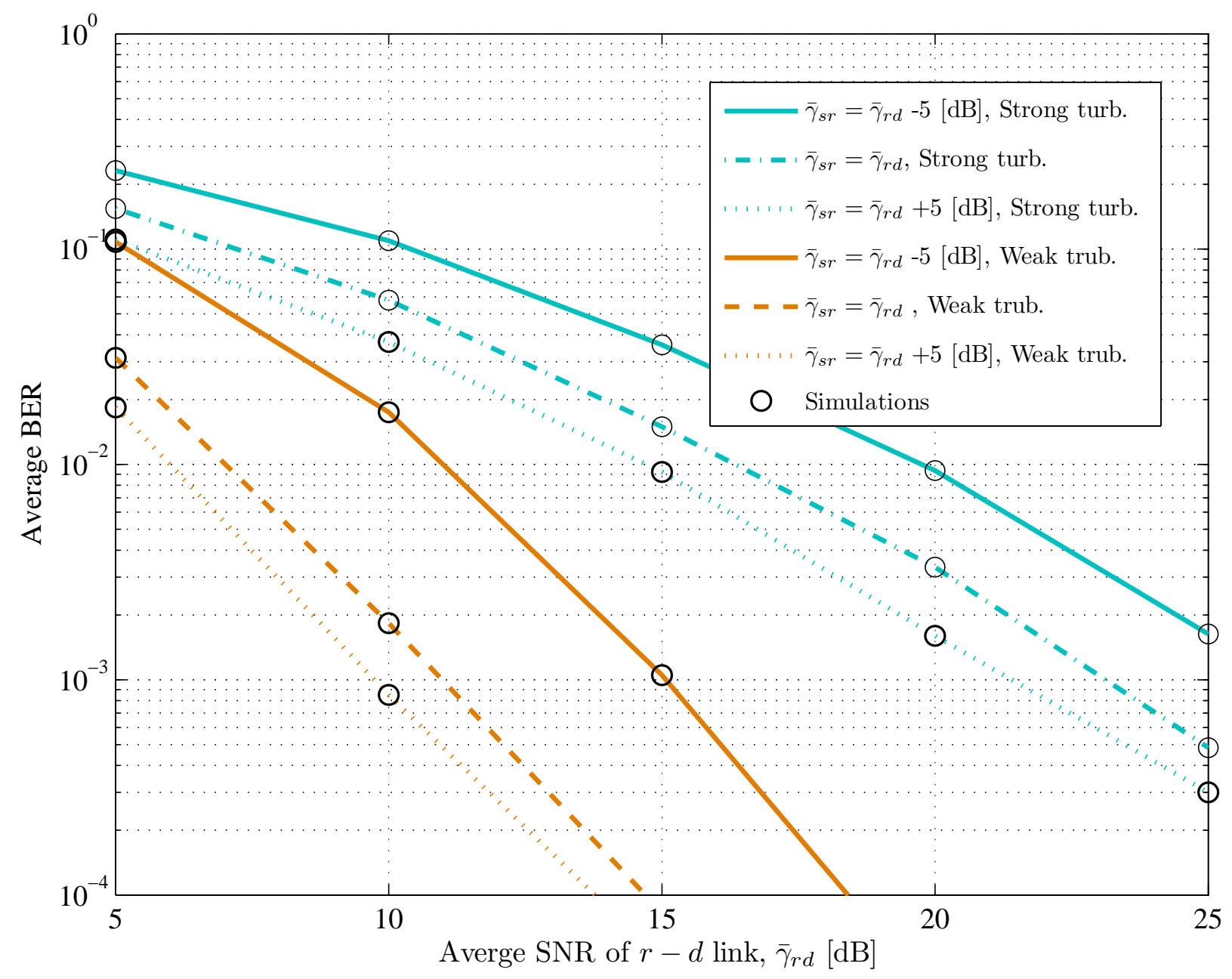

Fig. 6. The effect of non-identical $s-r$ and $r-d$ links on the BER.

Using (4) and (6), (20) can be written as,

$$
\begin{array}{r}
f_{X}(x)=\frac{\mu_{i}^{2}}{A_{0_{i}^{2}}^{\mu_{i}^{2}}} x^{\mu_{i}^{2}-1} \int_{\frac{x}{A_{0_{i}}}}^{\infty} h_{a t_{i}}-\mu_{i}^{2} \frac{\alpha_{i} \beta_{i}}{\eta_{i}}\left(\frac{h_{a t_{i}}}{\eta_{i}}\right)^{\beta_{i}-1} \exp \left[\left(\frac{h_{a t_{i}}}{\eta_{i}}\right)^{\beta}\right] \\
\times \underbrace{\left\{1-\exp \left[-\left(\frac{h_{a t_{i}}}{\eta_{i}}\right)^{\beta_{i}}\right]\right\}^{\alpha_{i}-1} d h_{a t_{i}} .}_{A 1}
\end{array}
$$

To solve the integration in (21), we expand the $A 1$ using the Newton's generalized binomial theorem i.e. $(1+y)^{t}=\sum_{j=0}^{\infty} \frac{\Gamma(t+1) y^{j}}{\Gamma(t-j+1) j !}$. After some mathematical manipulations (21) can be 
rewritten as,

$$
\begin{aligned}
f_{X}(x) & =\frac{\alpha_{i} \beta_{i} \mu_{i}^{2}}{\eta_{i}^{\beta_{i}} A_{0_{i}}^{\mu_{i}^{2}}} x^{\mu_{i}^{2}-1} \sum_{j=0}^{\infty} \frac{(-1)^{j} \Gamma\left(\alpha_{i}\right)}{j ! \Gamma\left(\alpha_{i}-j\right)} \\
& \times \int_{\frac{x}{A_{0_{i}}}}^{\infty} h_{a t_{i}}{ }^{\beta_{i}-1-\mu_{i}^{2}} \exp \left(\frac{-(j+1)}{\eta_{i}^{\beta_{i}}} h_{a t_{i}}^{\beta_{i}}\right) d h_{a t_{i}} .
\end{aligned}
$$

The PDF of the channel coefficient $\mathcal{H}_{i}$ can be deduced by evaluating the integral in (22) using [25, eq. 381.3], as

$$
f_{\mathcal{H}_{i}}(h)=\frac{\alpha_{i} \mu_{i}^{2}}{\left(\eta_{i} A_{0_{i}}\right)^{\mu_{i}^{2}}} \sum_{j=0}^{\infty} \Psi_{i}(j) h^{\mu_{i}^{2}-1} \Gamma\left[1-\frac{\mu_{i}^{2}}{\beta_{i}}, \frac{1+j}{\left(\eta_{i} A_{0_{i}}\right)^{\beta_{i}}} h^{\beta_{i}}\right], h \geq 0 .
$$

Now the PDF of instantaneous SNR $\gamma$ defined can easily be derived using [24, eq. (5-8)].

\section{APPENDIX II}

\section{VALIDATION OF PDF IN (9)}

For the expression in (23) to be a valid PDF it must be non-negative and area under this PDF should be unity. The non-negativity of PDF $f_{h}(h)$ in (23) can be observed from Table I as the range of $f_{\mathcal{H}_{i}}(h)$ in the possible domain of $h$ is positive.

TABLE I

\begin{tabular}{|l|l|l|l|l|l|l|l|}
\hline$h$ & 0 & 0.1 & 0.2 & 0.3 & 0.4 & 0.5 & 0.6 \\
\hline$f_{\mathcal{H}_{i}}(h)$ & 0 & .0284 & .0609 & .0948 & .1279 & .1547 & .1656 \\
\hline$h$ & 0.7 & 0.8 & 0.9 & 1.0 & 1.1 & 1.2 & 1.3 \\
\hline$f_{\mathcal{H}_{i}}(h)$ & .1513 & .1124 & .0645 & .0273 & .0082 & .0017 & .002 \\
\hline
\end{tabular}

As the PDF given in (4) is a valid PDF [11], area under this PDF should be one i.e. $\int_{0}^{\infty} f_{h_{a t_{i}}}\left(h_{a t_{i}}\right) d h_{a t_{i}}=1$. Rewriting the PDF $f_{h_{a t_{i}}}\left(h_{a t_{i}}\right)$ using Newton's generalized binomial theorem, we get

$$
\begin{aligned}
& \frac{\alpha_{i} \beta_{i}}{\eta_{i}} \sum_{j=0}^{\infty} \frac{(-1)^{j} \Gamma\left(\alpha_{i}\right)}{j ! \Gamma\left(\alpha_{i}-j\right)} \\
\times & \int_{0}^{\infty}\left(\frac{h_{a t_{i}}}{\eta_{i}}\right)^{\beta_{i}-1} \exp \left[-(1+j)\left(\frac{h_{a t_{i}}}{\eta_{i}}\right)^{\beta_{i}}\right] d h_{a t_{i}}=1 .
\end{aligned}
$$

After solving this integral, followed by some mathematical rearrangements (24) reduces to

$$
\sum_{j=0}^{\infty} \frac{(-1)^{j} \Gamma\left(\alpha_{i}\right)}{j ! \Gamma\left(\alpha_{i}-j\right)(1+j)}=\frac{1}{\alpha_{i}} .
$$


The area under the PDF $f_{\mathcal{H}_{i}}(h)$ is $\mathcal{S}=\int_{0}^{\infty} f_{\mathcal{H}_{i}}(h) d h$. Using (23) we get,

$$
\mathcal{S}=\frac{\alpha_{i} \mu^{2}}{\left(\eta_{i} A_{0}\right)^{\mu^{2}}} \sum_{j=0}^{\infty} \Psi(j) \int_{0}^{\infty} h^{\mu^{2}-1} \Gamma\left[1-\frac{\mu^{2}}{\beta_{i}}, \frac{1+j}{\left(\eta_{i} A_{0}\right)^{\beta_{i}}} h^{\beta_{i}}\right] d h .
$$

On substituting $h^{\beta_{i}}=r$ in (26), and using [25, eq. (6.455.1)], we get

$$
\mathcal{S}=\sum_{j=0}^{\infty} \frac{\Psi(j)}{(1+j)^{\frac{\mu^{2}}{\beta_{i}}}}{ }_{2} F_{1}\left(1,1 ; \frac{\mu^{2}}{\beta_{i}}+1 ; 0\right)
$$

where ${ }_{2} F_{1}(\cdot, \cdot ; \cdot ; \cdot)$ is Gauss hypergeometric function [26, eq. (07.23.02.0001.01)]. Now using [26, eq. (07.23.03.0001.01)] and (25) we get $\mathcal{S}=1$.

\section{REFERENCES}

[1] M. A. Kashani, M. M. Rad, M. Safari, and M. Uysal, "All-optical amplify-and-forward relaying system for atmospheric channels," IEEE Communications Letters, vol. 16, no. 10, pp. 1684-1687, Oct 2012.

[2] M. R. Bhatnagar,"Performance Analysis of Decode-and-Forward Relaying in Gamma-Gamma Fading Channels," IEEE Photonics Technology Letters, vol. 24, no. 7, pp. 545-547, Apr. 2012.

[3] P. K. Sharma, and P. Garg, "Bi-directional decode-XOR-forward relaying over $\mathcal{M}$-distributed free space optical links," IEEE Photonics Technology Letters, vol. 26, no. 19, pp. 1916-1919, Oct. 2014.

[4] L.C. Andrews, and R.L. Phillips, "Laser beam propagation through random media”, 2nd ed. Bellingham, Washington: SPIE Press, 2005.

[5] A. A. Farid, and S. Hranilovic, "Outage capacity optimization for free-space optical links with pointing errors," J. of Light. Tech., vol. 25, no. 7, pp. 1702-1710, July 2007.

[6] F. Yang, J. Cheng, and T. A. Tsiftsis, "Free-Space Optical Communication with Nonzero Boresight Pointing Errors," IEEE Trans. Commun., vol. 62, no. 2, pp. 713-725, Feb. 2014.

[7] F. Xu, M.A. Khalighi, P. Caussé, and S. Bourennane, "Channel coding and time-diversity for optical wireless links," Optics Express, vol. 17, no. 2, pp. 872-887, Jan. 2009.

[8] M.A. Khalighi, N. Schwartz, N. Aitamer, and S. Bourennane, "Fading reduction by aperture averaging and spatial diversity in optical wireless systems," IEEE/OSA Journal of Optical Communications and Networking, vol.1, no.6, pp. 580-593, Nov. 2009.

[9] F. S. Vetelino, C. Young, L. Andrews, and J. Recolons, "Aperture averaging effects on the probability density of irradiance fluctuations in moderate-to-strong turbulence," Appl. Opt. vol. 46, pp. 2099-2108, Mar. 2007.

[10] M. Safari and M. Uysal, "Relay-assisted free-space optical communication," IEEE Trans. Wireless Commun., vol. 7, pp. 5441-5449, Dec. 2008.

[11] R. Barrios and F. Dios, "Exponentiated Weibull distribution family under aperture averaging for Gaussian beam waves," Optics Express vol. 20, issue 12, pp. 13055-13064, May 2012.

[12] R. Barrios and F. Dios, "Reply to comment on The exponentiated Weibull distribution family under aperture averaging for Gaussian beam waves" Optics Express vol. 20, issue 12, pp. 20684-20687, Aug. 2012. 
[13] P. Wang, L. Zhang, L. Guo, F. Huang, T. Shang, R. Wang, and Y. Yang, “Average BER of subcarrier intensity modulated free space optical systems over the exponentiated Weibull fading channels," Optics Express vol. 22, issue 17, pp. 20828-20841, Aug. 2014.

[14] R. Barrios and F. Dios, "Probability of fade and BER performance of FSO links over the exponentiated Weibull fading channel under aperture averaging," in Unmanned/Unattended Sensors and Sensor Networks IX, ser. Proc. SPIE, vol. 8540, p. $85400 \mathrm{D}$, Oct. 2012.

[15] X. Yi, Z. Liu, and P. Yue, "Average BER of free-space optical systems in turbulent atmosphere with exponentiated Weibull distribution," Optics Letters, vol. 37, no. 24, pp. 5142-5144, Nov. 2012.

[16] M. Cheng, Y. Zhang, J. Gao, F. Wang, and F. Zhao, “Average capacity for optical wireless communication systems over exponentiated Weibull distribution non-Kolmogorov turbulent channels," Applied Optics, vol. 53, issue 18, pp. 4011-4017, Jun. 2014.

[17] P. K. Sharma, A. Bansal, P. Garg, T. A. Tsiftsis and R. Barrios, "Performance of FSO Links under Exponentiated Weibull Turbulence Fading with Misalignment Errors”, IEEE International Conference on Communications (ICC), pp.5110-5114, 8-12 June 2015, London, U.K..

[18] Xiang Yi and Mingwu Yao, "Free-space communications over exponentiated Weibull turbulence channels with nonzero boresight pointing errors," Opt. Express vol. 23, pp. 2904-2917, 2015.

[19] P. Wang, J. Zhang, L. Guo, T. Shang, T. Cao, R. Wang, and Y. Yang, "Performance Analysis for Relay-Aided Multihop BPPM FSO Communication System Over Exponentiated Weibull Fading Channels With Pointing Error Impairments,' IEEE Phot. Jour., vol.7, no.4, pp. 1-20, Aug. 2015.

[20] M. Niu, J. Cheng, J.F. Holzman, "Space-time coded MPSK coherent MIMO FSO systems in gamma-gamma turbulence," IEEE Wireless Communications and Networking Conference (WCNC), pp.4266-4271, 7-10 April 2013.

[21] P. K. Sharma, A. Bansal, and P. Garg, "Relay Assisted Bi-directional Communication in Generalized Turbulence Fading," Journal of Lightwave Technology, vol. 33, no. 1, pp. 133-139, Jan. 2015.

[22] L. C. Andrews, "Aperture-averaging factor for optical scintillations of plane and spherical waves in the atmosphere," J. Opt. Soc. Am., vol. 9, no. 4, pp. 597- 600, Apr. 1992.

[23] R. Barrios, F. Dios, "Exponentiated Weibull model for the irradiance probability density function of a laser beam propagating through atmospheric turbulence",Optics \& Laser Technology, Vol. 45, pp. 13-20, February 2013.

[24] A. Papoulis and S. U. Pillai, "Probability, random variables and stochastic processes",4th edition , Tata McGraw Hill, New Delhi, 2002.

[25] I. S. Gradshetyn and I. M. Ryzhic, Table of integrals, series and products, (U.S.A.: Academic Press, 6th ed.), 2000.

[26] I. Wolfram Research, Mathematica Edition: Version 8.0, Champaign, Illinois:Wolfram Research Inc., 2010.

[27] A.P. Prudnikov, Yu. A. Brychkov and O. I. Marichov, Integrals and Series: More Special Functions, (Gordon and Breach Science Publishers), vol. 3, 1990.

[28] A. Muller, and J. Speidel, "Exact symbol error probability of m-psk for multihop transmission with regenerative relays," IEEE Communications Letters, vol.11, no.12, pp.952,954, December 2007.

[29] P. K. Sharma, and P. Garg "Performance Analysis of Full Duplex Decode and Forward Cooperative Relaying over Nakagami-m Fading Channels", Wiley Transactions on Emerging Telecommunication Technologies, vol. 25, no. 9, pp. 905-913, Sept. 2014.

[30] M. R. McKay, A. Zanella, I. B. Collings, and M. Chiani, "Error probability and SINR analysis of optimum combining in rician fading," IEEE Transactions on Communications, vol. 57, no. 3, pp. 676-687, March 2009. 
[31] R. Barios, "Exponentiated Weibull fading channel model in free-space optical communications under atmospheric turbulence," Ph.D. dissertation submitted to Universitat Polytecnica de Catalunya, Barcelona, Spain, April 2013. 